\title{
The 9-A solution: How mRNA pseudoknots promote efficient programmed -1 ribosomal frameshifting
}

\author{
EWAN P. PLANT, ${ }^{1}$ KRISTI L. MULDOON JACOBS, ${ }^{2}$ JASON W. HARGER, ${ }^{2}$ ARTURAS MESKAUSKAS, ${ }^{1}$ \\ JONATHAN L. JACOBS, ${ }^{1}$ JENNIFER L. BAXTER, ${ }^{1}$ ALEXEY N. PETROV, ${ }^{1}$ and JONATHAN D. DINMAN ${ }^{1}$ \\ ${ }^{1}$ Department of Cell Biology and Molecular Genetics, University of Maryland, College Park, Maryland 20742, USA \\ ${ }^{2}$ Department of Molecular Genetics and Microbiology, and The Graduate Programs in and Molecular Bioscience Rutgers/University of \\ Medicine and Dentistry of New Jersey, Robert Wood Johnson Medical School, Piscataway, New Jersey 08854, USA
}

\begin{abstract}
There is something special about mRNA pseudoknots that allows them to elicit efficient levels of programmed -1 ribosomal frameshifting. Here, we present a synthesis of recent crystallographic, molecular, biochemical, and genetic studies to explain this property. Movement of $9 \AA$ by the anticodon loop of the aminoacyl-tRNA at the accommodation step normally pulls the downstream mRNA a similar distance along with it. We suggest that the downstream mRNA pseudoknot provides resistance to this movement by becoming wedged into the entrance of the ribosomal mRNA tunnel. These two opposing forces result in the creation of a local region of tension in the mRNA between the A-site codon and the mRNA pseudoknot. This can be relieved by one of two mechanisms; unwinding the pseudoknot, allowing the downstream region to move forward, or by slippage of the proximal region of the mRNA backwards by one base. The observed result of the latter mechanism is a net shift of reading frame by one base in the $5^{\prime}$ direction, that is, a -1 ribosomal frameshift.
\end{abstract}

Keywords: Virus; ribosome; translation; genetic code; recoding; structure/function

After a generation spent in the shadows, the ribosome is enjoying a renaissance. Recent breakthroughs in X-ray crystallography and cryoelectron microscopy have given us atomic-level views of this complex molecular machine (Ban et al. 2000; Wimberly et al. 2000; Harms et al. 2001; Spahn et al. 2001; Yusupov et al. 2001) that are bringing into focus the relationship between ribosome structure and function (Gabashvili et al. 1999; Agrawal et al. 2000; Carter et al. 2000; Frank and Agrawal 2000; Mueller et al. 2000; Nissen et al. 2000; Schluenzen et al. 2000; Beckmann et al. 2001; Nissen et al. 2001; Pioletti et al. 2001; Polacek et al. 2001; Thompson et al. 2001; Yusupova et al. 2001; Noller et al. 2002; Schmeing et al. 2002; Simonson and Lake 2002). One of the major requirements of the ribosome is to maintain translational reading frame, and an increasing number of cis-acting mRNA signals that alter this have been used to probe this essential function of the translational machinery. These translational "recoding" events (Gesteland and Atkins 1996) can take many forms, for example, "slips" of one

Reprint requests to: Jonathan D. Dinman, Department of Cell Biology and Molecular Genetics, Microbiology Bldg. Rm. 2135, University of Maryland, College Park, MD 20742, USA; e-mail: jd280@umail.umd.edu. Article and publication are at http://www.rnajournal.org/cgi/doi/ 10.1261/rna.2132503. or more bases, "hops" spanning as many as 50 nucleotides, and "shunts" around large mRNA secondary structures (for review, see Jacks 1990; Brierley 1995; Farabaugh 1996; Giedroc et al. 2000). Programmed -1 ribosomal frameshifting $(-1 \mathrm{PRF})$ is the most widely used translational recoding mechanism of RNA viruses. The -1 PRF signal can be broken down into three discrete parts: the "slippery site", a linker region, and a downstream region of secondary mRNA structure, typically an mRNA pseudoknot. Mutagenesis studies from many different laboratories have demonstrated that the primary sequence of the slippery site and its placement in relation to the incoming translational reading frame is critical: It must be X XXY YYZ, where X must be a stretch of three identical nucleotides, $\mathrm{Y}$ is either AAA or UUU, and Z is A, C, or U. Although less is known about the linker region, its length and base composition vary, and these parameters are thought to be important for determining the extent of -1 PRF in a virus-specific manner (Brierley et al. 1992; Dinman and Wickner 1992; Morikawa and Bishop 1992; Kollmus et al. 1994; Kim et al. 1999; Bertrand et al. 2002). Modeling of the MMTV frameshift signal into the Escherichia coli ribosome by Giedroc et al. (2000) supports the notion that the linker region positions the pseudoknot on the surface of the ribosome when the A- and $\mathrm{P}$-sites are occupied by the slippery site sequence. The func- 
tion of the downstream secondary structure is to induce elongating ribosomes to pause (Rice et al. 1985), and its ability to induce ribosomal pausing is critical for efficient -1 PRF to occur ( $\mathrm{Tu}$ et al. 1992; Somogyi et al. 1993; Lopinski et al. 2000; Kontos et al. 2001). The generally accepted mechanism of -1 PRF is as follows: (1) The secondary mRNA structure forces elongating ribosomes to pause, and the length of the linker is such that the ribosomal A- and P-site bound aminoacyl- (aa-) and peptidyl-tRNAs are positioned over the slippery site; (2) the sequence of the slippery site allows for re-pairing of the tRNAs to the -1 frame codons after they "simultaneously slip" by one base in the $5^{\prime}$ direction along the mRNA; and (3) subsequent melting of the downstream mRNA secondary structure allows the ribosome to continue elongation of the nascent polypeptide in the new translational reading frame. This is diagrammed in Figure 1.
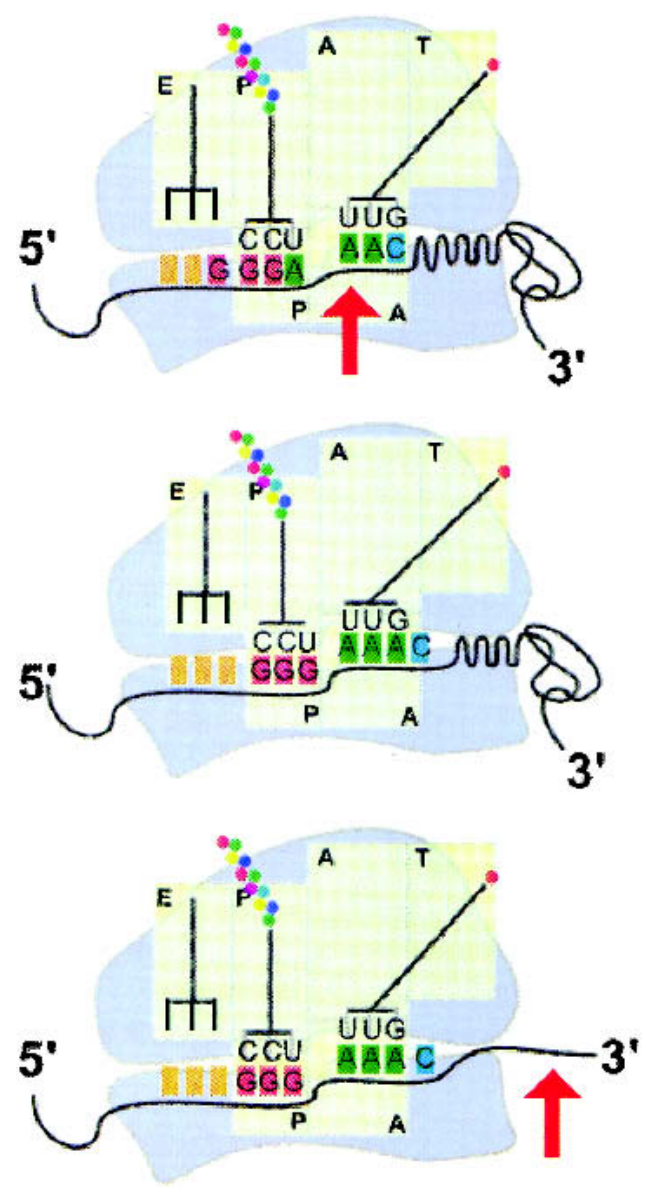

FIGURE 1. Overview of programmed -1 ribosomal frameshifting. (Top) An mRNA pseudoknot induces elongating ribosomes to pause with their A- and P-site tRNAs positioned over the heptameric X XXY YYZ "slippery site" (red arrow). The incoming frame is indicated by spaces. (Middle) While paused at the slippery site, if the ribosome shifts by 1 base in the $5^{\prime}$ direction, the non-wobble bases of both the A- and P-site tRNAs can re-pair with the new -1 frame codons. (Bottom) The mRNA pseudoknot is denatured (arrow), and elongation continues in new reading frame.

\section{Downstream elements}

mRNA pseudoknots have been shown to increase -1 PRF efficiencies significantly more than comparable stem-loop structures (for review, see Brierley 1995). Though some examples exist in the literature of simple stem-loops that promote efficient $-1 \mathrm{PRF}$, recent findings in which two previously suspected stem-loops were in fact more complex structures suggest that mRNA pseudoknots are universally required to promote efficient levels of -1 PRF (Barry and Miller 2002; Dinman et al. 2002). One of the main questions in the field is how mRNA pseudoknots promote efficient levels of -1 PRF. A major consideration of many models of -1 PRF has been that the discontinuous nature of pseudoknot structures, and the poorly matched geometry between the pseudoknot and the ribosomal helicase, may result in the pseudoknot blocking the entry of the mRNA into the mRNA tunnel (Yusupova et al. 2001; Dinman et al. 2002). Accordingly, it has been proposed that mRNA pseudoknots might induce ribosomes to pause at the frameshift signal for longer times, and/or better direct them to pause with the A- and P-site tRNAs optimally positioned over the slippery site. Both of these mechanisms would increase the likelihood of frameshifting. However, a recent study using mRNA heelprinting techniques showed that there were no differences in either the extent or duration of ribosome pausing promoted by a stem-loop compared to an mRNA pseudoknot (Kontos et al. 2001). Thus, it is clear that ribosome pausing is necessary but not sufficient to promote efficient -1 PRF.

\section{The 'integrated model' of PRF: Timing is everything}

Integration of the model describing -1 PRF into the context of the "hybrid states" model of the translation elongation cycle (Moazed and Noller 1989; Noller et al. 2002) places the -1 PRF event after delivery of aa-tRNA to the ribosome but prior to translocation (Harger et al. 2002). A "co-translocational" model of -1 PRF suggests that, as a poor substrate for the ribosomal helicase, the mRNA pseudoknot blocks entry of the mRNA into the downstream tunnel, inhibiting accurate eEF-2 (the eukaryotic homolog of EF$\mathrm{G})$-catalyzed translocation, resulting in translocation events of two rather than three nucleotides (Weiss et al. 1989; Yusupova et al. 2001). However, all of the genetic and pharmacological evidence to date shows that alteration of eEF-2 function has no effect on -1 PRF, suggesting that postpeptidyltransfer ribosomes cannot slip (Tumer et al. 1998; Harger et al. 2001; Hudak et al. 2001). This conclusion is further supported by a biochemical study showing that ribosomes containing an acetylated aa-tRNA in the A-site (analogous to a post-peptidyltransfer A/A hybrid state tRNA) maintain frame most accurately (Fredrick and Noller 2002). The argument against slippage after peptidyltransfer is also reasonable from a bioenergetics viewpoint: Whereas prior to peptidyltransfer, the ribosome has not 
irrevocably committed the nascent peptide to a specific new amino acid or to a particular reading frame, the formation of a covalent bond at peptidyltransfer marks a thermodynamic "point of no return" committing ribosomes to a specific amino acid. Thus, it is energetically more favorable for error correction (e.g., aa-tRNA selection, reading frame maintenance, etc.) to occur before, rather than after this step. Thus, a refinement of the integrated model places the -1 PRF event after delivery of aa-tRNA to the ribosome but prior to peptidyltransfer (Harger et al. 2002).

\section{Energy requirements}

An attractive feature of the co-translocational model is that it purports that slippage occurs concurrently with ribosome movement. Implicit in this model is that hydrolysis of GTP by eEF-2 provides the energy required to effect the frameshift. Energy is also added into the system earlier in the elongation cycle when GTP is hydrolyzed by eEF1A (the eukaryotic EF-Tu homolog) during accommodation of the aa-tRNA from the hybrid A/T to A/A states. Hydrolysis of GTP $\rightarrow$ GDP releases approximately $-7.3 \mathrm{kcal} / \mathrm{mole}$; that is, this much energy is added to the system. Coincidentally, unpairing of aa- and peptidyl-tRNAs from the 0 -frame and their subsequent re-pairing to the -1 frame codons result in the net loss of up to 4 or 5 hydrogen bonds (depending on the identities of the 0 -frame wobble bases), a gain of 6.4-8.0 $\mathrm{kcal} /$ mole (taking a value of $-1.6 \mathrm{kcal} / \mathrm{mole}$ per $\mathrm{H}$-bond at physiological $\mathrm{pH}$ ). The approximate equivalence of the energies added into and subtracted from the system suggests that GTP hydrolysis at this step adds enough energy into the 0 -frame state to effectively lower the threshold for slippage into the -1 frame state.

\section{The 9-Å solution}

Significant movement of the aa-tRNA also occurs during accommodation from the A/T to the A/A state. Most importantly with regard to this discussion, the A-site anticodon loop moves approximately $9 \AA$ during this transition (for review, see Noller et al. 2002). This movement is predicted from differences in the A/A state structure derived from X-ray crystallography and the A/T hybrid state modeled using a rigid tRNA constrained by chemical footprinting and directed hydroxyl radical probing experiments. Interestingly, the empirically determined average distance between two adjacent bases along an mRNA in the ribosomal tunnel is $5.2 \AA$ (Yusupova et al. 2001). Since the anticodon is base-paired to the mRNA, a priori the mRNA must also be pulled along with it by $9 \AA$ in the $5^{\prime}$ direction. Whether or not all of the mRNA movement is in the $5^{\prime}$ direction, the observation that one additional base becomes inaccessible to reverse transcriptase in a ribosome "toeprint" assay upon addition of an aa-tRNA into the A-site supports the general idea of 5' mRNA movement concomitant with accommodation (Fredrick and Noller 2002).
Docking of the mRNA pseudoknot crystal structure from Beet Western Yellows Virus -1 PRF signal (Su et al. 1999; Egli et al. 2002) into the context of the path of the mRNA through the ribosome (Fig. 2; Yusupova et al. 2001; Ogle et al. 2001) reveals that the $37-\AA \times 18-\AA$ face of the pseudoknot is significantly greater than the entrance of the ribosomes' mRNA downstream tunnel, which quickly tapers to $17 \AA \times 20 \AA$ between the S3, S4, and S5 proteins (Fig. 3). Thus, if movement of the immediate region of the mRNA $3^{\prime}$ of the P- and A-site positioned slippery site is blocked by the inability of the mRNA pseudoknot to enter the downstream tunnel, then movement of the A-site codon by one base in the $5^{\prime}$ direction would stretch the intervening region of the mRNA, creating an area of local tension along it between the A-site codon and the mRNA pseudoknot. This would require a certain degree of elasticity by the mRNA linker region, an idea that has been experimentally validated in single-molecule experiments (Bustamante et al. 2000). In addition, upon accommodation of the aa-tRNA into the A/A hybrid state, the movement of the small subunit rRNA "530 loop" further serves to lock the aa-tRNA into position on the ribosome (Carter et al. 2000), preventing the aatRNA from moving back. This scenario would find the linker region of the mRNA between the Scylla of the mRNA pseudoknot and the Charybdis of the ribosome. There are three possible solutions for relieving the resulting tension: (1) unwinding the pseudoknot and allowing the slippery site distal region of the mRNA to move forward by one base; (2) uncoupling of the A- and P-site codon:anticodon interactions, allowing slippage of this local region of mRNA by one base in the $3^{\prime}$ direction, and re-pairing in the -1 frame; or (3) ribosome dissociation and aborted translation. The frameshift scenario is modeled in Figure 4 . In the sec-

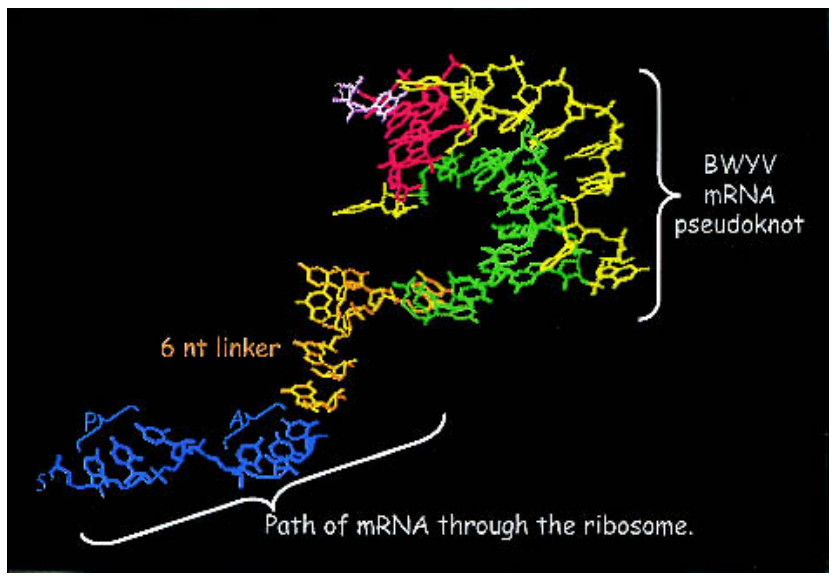

FIGURE 2. The mRNA pseudoknot from Beet Western Yellows Virus (Egli et al. 2002). Stem 1 residues are depicted in green, loops and bulging residues in yellow, stem 2 residues are shown in red, and the $3^{\prime}$ base is colored lavender. We attached a 6-nt linker RNA (orange) and $\mathrm{A}$ - and $\mathrm{P}$-site codons (blue) modeled to fit into the downstream mRNA tunnel of the Thermus thermophilis 30S ribosomal subunit (Yusupova et al. 2001). Figure constructed using the Swiss-Pdb Viewer. 

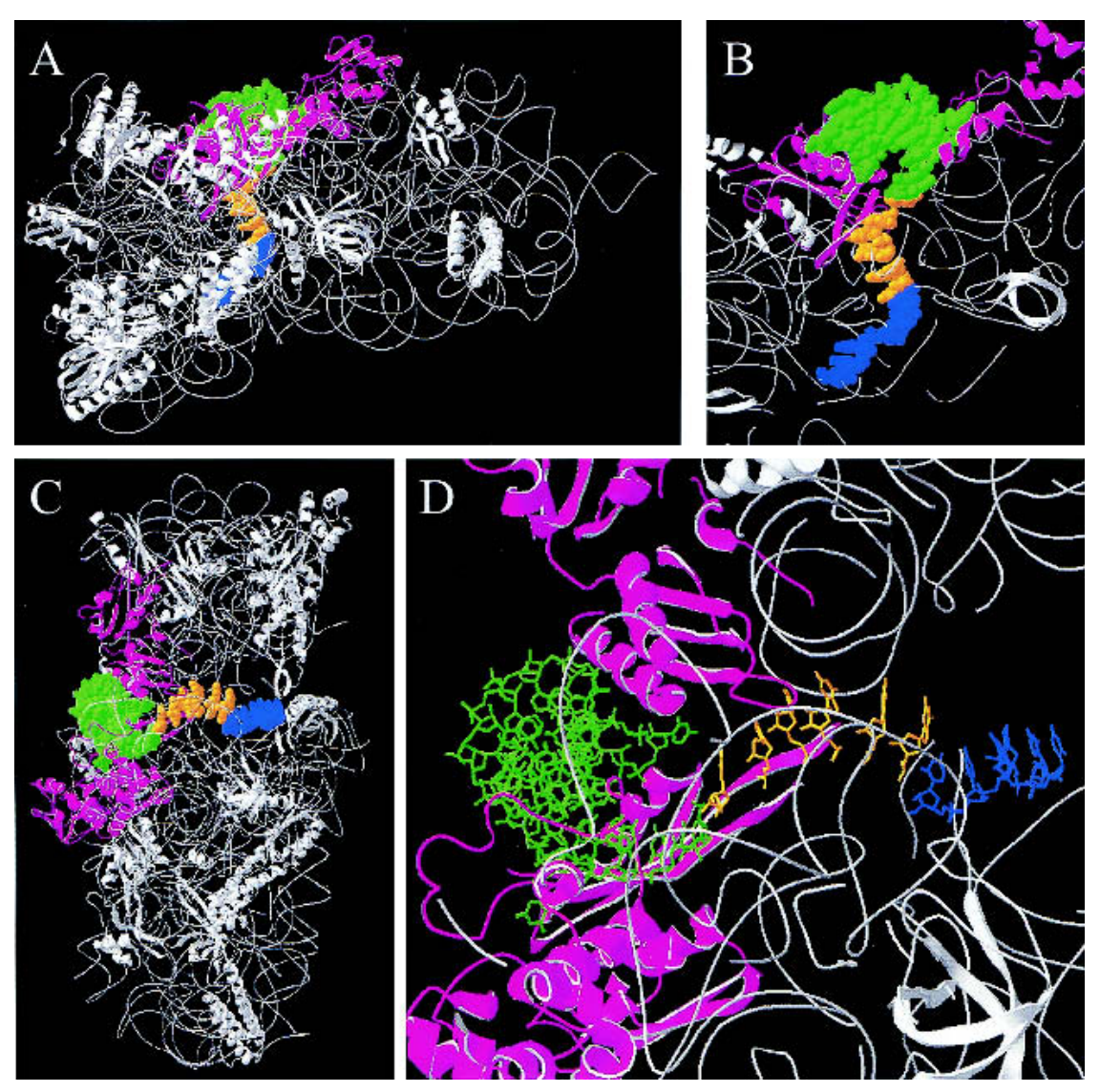

FIGURE 3. Docking of the mRNA pseudoknot crystal structure from the Beet Western Yellows Virus -1 PRF signal into the mRNA tunnel of the Thermus thermophilis 30S subunit (Ogle et al. 2001; Yusupova et al. 2001). The rRNA is shown as a gray ribbon; ribosomal proteins are white except for S3, S4, and S5, which are pink; the BWYV mRNA pseudoknot is green; the 6-nt linker in the mRNA tunnel is orange; the A- and P-site codons are shown as blue. Figures were constructed using the Swiss-Pdb Viewer. (A) View from the intersubunit side of the $30 \mathrm{~S}$ subunit. S3 is to the left and behind the mRNA pseudoknot, S4 is at the top right, and S5 is partially obscured by rRNA at the middle right. (B) 50 - $\AA$ cutaway section with S3 and S5 to the left, and S4 to the right. (C) View from shoulder side of $30 \mathrm{~S}$ subunit. S3 is on the top, S4 is on the bottom, and $\mathrm{S} 5$ is behind the pseudoknot. (D) 50- $\AA$ cutaway section of view shown in panel $C$.

ond scenario, since mRNA slippage is energetically favored before peptidyltransfer, it stands to reason that the slip must occur either concurrent with the A/T to A/A transition, or shortly thereafter, that is, before peptidyltransfer. In support of this model, the notion of mRNA movement inside of the ribosome has also been invoked to explain the programmed +1 ribosomal frameshift of the yeast Ty3 retrotransposable element (Stahl et al. 2002).

\section{Supporting data}

This model is consistent with all of the findings to date. For example, we find that inhibition of peptidyltransfer promotes increased efficiencies of -1 PRF (Dinman et al. 1997; A. Meskauskas and J. Dinman, in prep.). We hypothesize that this would increase the amount of time that a ribosome is paused over the frameshift signal with both its A- and P-sites occupied by tRNAs, increasing the likelihood of the
-1 slip to occur in the most energetically favorable window. The model also predicts that reducing or increasing the linker region by a single nucleotide would affect the balance of forces between mRNA slippage and mRNA pseudoknot unwinding. This in turn would impact on frameshifting efficiencies. Direct experiments to test this are consistent with this prediction (Napthine et al. 1999), as are results obtained with residue swapping mutants at the base of stem 1 (Kim et al. 1999; Nixon et al. 2002). Eukaryotic ribosomes also contain homologs for the three bacterial proteins mapped to the entrance of the mRNA tunnel. Specifically, the eukaryotic homolog of prokaryotic S3 is eS3, that of $\mathrm{S} 4$ is eS9, and that of S5 is eS2. Of particular note, a semidominant mutant of the yeast S4 homolog, SUP46, was found to slightly enhance -1 PRF in yeast (Dinman and Wickner 1994). The model also explains the inability of the heelprint analysis to detect differences between mRNA pseudoknot- and stemloop-induced ribosome pausing. The heelprint analysis maps the $5^{\prime}$ end of the mRNA fragment protected by the ribosome from nuclease hydrolysis; that is, it should detect differences in the position of the protected fragment in the case of ribosome slippage. However, since this model posits that slippage actually involves only a small region of mRNA inside of the mRNA tunnel rather than any gross movement by the ribosome, there should be no difference in the position of the $5^{\prime}$ end of the protected fragment by this method. Unfortunately, although the authors of that study determined the size of the ribosome-protected fragment using a pseudoknot-containing mRNA, comparison with a similar stem-loop-containing substrate, which may have revealed a difference in protection at the $3^{\prime}$ end, was not assayed (Kontos et al. 2001). It will also be interesting to determine whether such a difference can be detected by mRNA "toeprinting", which maps the $3^{\prime}$ end of ribosome pause events (Kozak 1998; Pestova et al. 1998; Wang et al. 1999). However, the presence of the mRNA pseudoknot may also prevent the detection of any such changes.

\section{Unfinished business}

We have presented a model based on new structural and biochemical data that explains how mRNA pseudoknots 


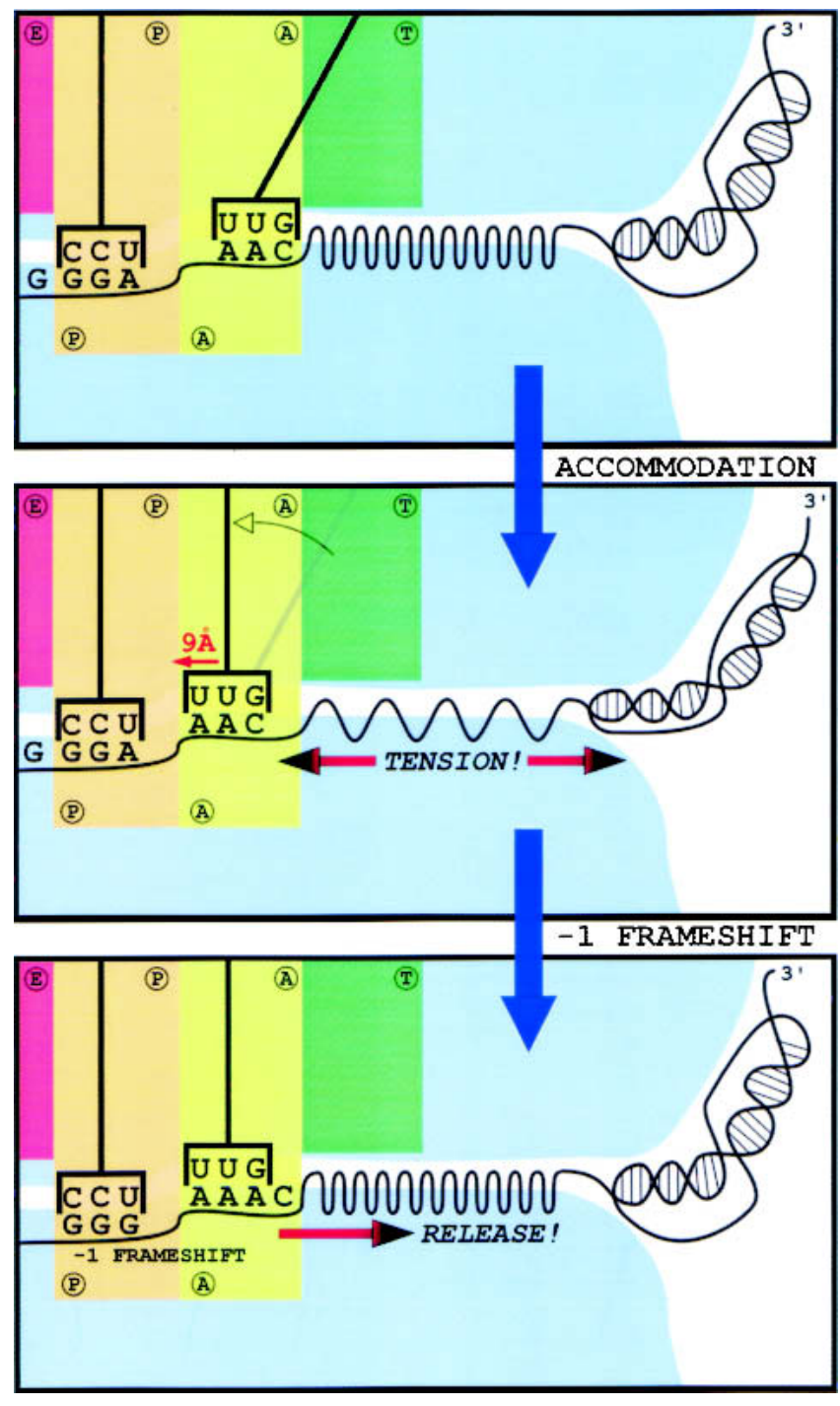

FIGURE 4. The $9-\AA$ solution. (Top) The 0 -frame A- and P-site codons of a programmed -1 ribosomal frameshift signal are basepaired to cognate peptidyl- and aa-tRNAs occupying the P/P and A/T hybrid states respectively. (Middle) Upon accommodation, the anticodon loop of the aa-tRNA moves $9 \AA$ in the $5^{\prime}$ direction, pulling the $3^{\prime}$ mRNA sequence along with it. The mRNA pseudoknot is too large to enter the downstream tunnel, with the consequence that the linker region between the A-site codon and the mRNA pseudoknot is stretched, creating a localized region of tension in the linker mRNA. (Bottom) Decoupling of the A- and P-site codon:anticodon interactions from the 0 -frame, and re-pairing in the -1 frame repositions the mRNA so as to relieve the tension.

specifically enhance programmed -1 ribosomal frameshifting. It must be stressed, however, that here we have modeled a eukaryotic -1 PRF signal into an archaebacterial ribosome. Though these classes of frameshift signals have not been tested in archae, the process is not mRNA pseudoknot-specific in E. coli (for review, see Farabaugh 1996). However, given the structural and functional conservation of the core elements of the ribosome, we believe that the modeling approach taken here is generally valid. It is also important to bear in mind that this is merely a hypothesis that remains to be tested empirically. One possible approach might involve experiments designed to measure distances between nucleotides in the linker region before and after aa-tRNA accommodation. An alternative strategy could entail altering the rotational freedom of the mRNA pseudoknot such that repositioning would allow more or less of the linker mRNA to enter the mRNA tunnel, thereby decreasing or increasing the extent of the tension that this stretch of mRNA would be subjected to after accommodation. If the model is correct, 'ribosomal frameshifting' itself would be a misnomer because it is actually a very local region of mRNA that slips. Though this model may answer some important questions, it gives rise to many more, some of which may be answered by better understanding the differences between pseudoknots that enhance frameshifting and those that do not (Wang et al. 2002). Are the dimensions of a pseudoknot at the point where triplex base pairing occurs, providing some steric hindrance to the progression of the mRNA into the entry tunnel compared to the ability of the ribosome to accommodate stem-loop structures? Is progression of the mRNA delayed due to the discontinuous nature of the pseudoknot helices being more difficult to unwind than the continuous helix of a stem loop? Alternatively, does the systematic unwinding of a pseudoknot provide some torsional constraint (Dinman 1995; Dinman et al. 2002) that hinders the progression of the mRNA? It is not known whether any of these events occur through specific interactions with the ribosome (Kontos et al. 2001), or in concert with a trans-acting factor (Dinman et al. 2002), or due to the intrinsic nature of the pseudoknot. There are also unanswered questions surrounding the linker region. For example, do its length and base composition vary as a means of fine-tuning frameshift efficiencies between different virus-host systems? Further, does the shape of the mRNA tunnel play a role in how the mRNA is stretched? Although there are no crystal structures of ribosomes containing peptidyl-tRNA without aa-tRNA, presumably such structures could show the accessibility of the A-site codon to an eEF-2-bound tRNA, perhaps explaining the need for a 9-A movement upon accommodation during normal translation. Finally, are there speciesspecific interactions between the proteins at the entrance of the mRNA tunnel and mRNA pseudoknots of different viruses? As we gather more information about the atomicscale structures of ribosomes from different species, -1 PRF will continue to provide us with a powerful tool to gain more insight into the relationship between ribosome structure and function.

\section{ACKNOWLEDGMENTS}

This work was supported by a grant to J.D.D. from the NIH (GM58859). J.L.B. was supported in part by a training grant from the NIH (T32 AI 51967). 


\section{REFERENCES}

Agrawal, R.K., Spahn, C.M., Penczek, P., Grassucci, R.A., Nierhaus, K.H., and Frank, J. 2000. Visualization of tRNA movements on the Escherichia coli $70 \mathrm{~S}$ ribosome during the elongation cycle. J. Cell Biol. 150: 447-460.

Ban, N., Nissen, P., Hansen, J., Moore, P.B., and Steitz, T.A. 2000. The complete atomic structure of the large ribosomal subunit at $2.4 \AA$ resolution. Science 289: 905-920.

Barry, J.K. and Miller, W.A. 2002. A -1 ribosomal frameshift element that requires base pairing across four kilobases suggests a novel mechanism of regulating ribosome and replicase traffic on a viral RNA. Proc. Natl. Acad. Sci. 99: 11133-11138.

Beckmann, R., Spahn, C.M., Eswar, N., Helmers, J., Penczek, P.A., Sali, A., Frank, J., and Blobel, G. 2001. Architecture of the proteinconducting channel associated with the translating $80 \mathrm{~S}$ ribosome. Cell 107: 361-372.

Bertrand, C., Prere, M.F., Gesteland, R.F., Atkins, J.F., and Fayet, O. 2002. Influence of the stacking potential of the base $3^{\prime}$ of tandem shift codons on -1 ribosomal frameshifting used for gene expression. RNA 8: 16-28.

Brierley, I. 1995. Ribosomal frameshifting on viral RNAs. J. Gen. Virol. 76: $1885-1892$.

Brierley, I.A., Jenner, A.J., and Inglis, S.C. 1992. Mutational analysis of the 'slippery sequence' component of a coronavirus ribosomal frameshifting signal. J. Mol. Biol. 227: 463-479.

Bustamante, C., Smith, S.B., Liphardt, J., and Smith, D. 2000. Singlemolecule studies of DNA mechanics. Curr. Opin. Struct. Biol. 10: $279-285$

Carter, A.P., Clemons, W.M., Brodersen, D.E., Morgan-Warren, R.J., Wimberly, B.T., and Ramakrishnan, V. 2000. Functional insights from the structure of the $30 \mathrm{~S}$ ribosomal subunit and its interactions with antibiotics. Nature 407: 340-348.

Dinman, J.D. 1995. Ribosomal frameshifting in yeast viruses. Yeast 11: 1115-1127.

Dinman, J.D. and Wickner, R.B. 1992. Ribosomal frameshifting efficiency and Gag/Gag-pol ratio are critical for yeast $M$ doublestranded RNA virus propagation. J. Virol. 66: 3669-3676.

Dinman, J.D. and Wickner, R.B. 1994. Translational maintenance of frame: Mutants of Saccharomyces cerevisiae with altered -1 ribosomal frameshifting efficiencies. Genetics 136: 75-86.

Dinman, J.D., Ruiz-Echevarria, M.J., Czaplinski, K., and Peltz, S.W. 1997. Peptidyl transferase inhibitors have antiviral properties by altering programmed -1 ribosomal frameshifting efficiencies: Development of model systems. Proc. Natl. Acad. Sci. 94: 6606-6611.

Dinman, J.D., Richter, S., Plant, E.P., Taylor, R.C., Hammell, A.B., and Rana, T.M. 2002. The frameshift signal of HIV-1 involves a potential intramolecular triplex RNA structure. Proc. Natl. Acad. Sci. 99: 5331-5336.

Egli, M., Minasov, G., Su, L., and Rich, A. 2002. Metal ions and flexibility in a viral RNA pseudoknot at atomic resolution. Proc. Natl. Acad. Sci. 99: 4302-4307.

Farabaugh, P.J. 1996. Programmed translational frameshifting. Microbiol. Rev. 60: 103-134.

Frank, J. and Agrawal, R.K. 2000. A ratchet-like inter-subunit reorganization of the ribosome during translocation. Nature 406: 318322.

Fredrick, K. and Noller, H.F. 2002. Accurate translocation of mRNA by the ribosome requires a peptidyl group or its analog on the tRNA moving into the 30S P Site. Mol. Cell 9: 1125-1131.

Gabashvili, I.S., Agrawal, R.K., Grassucci, R., Squires, C.L., Dahlberg, A.E., and Frank, J. 1999. Major rearrangements in the 70S ribosomal 3D structure caused by a conformational switch in $16 \mathrm{~S}$ ribosomal RNA. EMBO J. 18: 6501-6507.

Gesteland, R.F. and Atkins, J.F. 1996. Recoding: Dynamic reprogramming of translation. Annu. Rev. Biochem. 65: 741-768.

Giedroc, D.P., Theimer, C.A., and Nixon, P.L. 2000. Structure, stability and function of RNA pseudoknots involved in stimulating ribosomal frameshifting. J. Mol. Biol. 298: 167-185.
Harger, J.W., Meskauskas, A., Nielsen, N., Justice, M.C., and Dinman, J.D. 2001. TyT1 retrotransposition and programmed +1 ribosomal frameshifting require the integrity of the protein synthetic translocation step. Virology 286: 216-224.

Harger, J.W., Meskauskas, A., and Dinman, J.D. 2002. An 'integrated model' of programmed ribosomal frameshifting and post-transcriptional surveillance. Trends Biochem. Sci. 27: 448-454.

Harms, J., Schluenzen, F., Zarivach, R., Bashan, A., Gat, S., Agmon, I., Bartels, H., Franceschi, F., and Yonath, A. 2001. High resolution structure of the large ribosomal subunit from a mesophilic eubacterium. Cell 107: 679-688.

Hudak, K.A., Hammell, A.B., Yasenchak, J., Tumer, N.E., and Dinman, J.D. 2001. A C-terminal deletion mutant of pokeweed antiviral protein inhibits programmed +1 ribosomal frameshifting and Ty1 retrotransposition without depurinating the sarcin/ricin loop of rRNA. Virology 279: 292-301.

Jacks, T. 1990. Translational suppression in gene expression in retroviruses and retrotransposons. Curr. Top. Microbiol. Immunol. 157: 93-124.

Kim, Y.G., Su, L., Maas, S., O’Neill, A., and Rich, A. 1999. Specific mutations in a viral RNA pseudoknot drastically change ribosomal frameshifting efficiency. Proc. Natl. Acad. Sci. 96: 14234-14239.

Kollmus, H., Honigman, A., Panet, A., and Hauser, H. 1994. The sequences of and distance between two cis-acting signals determine the efficiency of ribosomal frameshifting in human immunodeficiency virus type 1 and human T-cell leukemia virus type II in vivo. J. Virol. 68: 6087-6091.

Kontos, H., Napthine, S., and Brierley, I. 2001. Ribosomal pausing at a frameshifter RNA pseudoknot is sensitive to reading phase but shows little correlation with frameshift efficiency. Mol. Cell Biol. 21: 8657-8670.

Kozak, M. 1998. Primer extension analysis of eukaryotic ribosomemRNA complexes. Nucleic Acids Res. 26: 4853-4859.

Lopinski, J.D., Dinman, J.D., and Bruenn, J.A. 2000. Kinetics of ribosomal pausing during programmed -1 translational frameshifting. Mol. Cell Biol. 20: 1095-1103.

Moazed, D. and Noller, H.F. 1989. Intermediate states in the movement of transfer RNA in the ribosome. Nature 342: 142-148.

Morikawa, S. and Bishop, D.H.L. 1992. Identification and analysis of the gag-pol ribosomal frameshift site of feline immunodeficiency virus. Virology 186: 389-397.

Mueller, F., Sommer, I., Baranov, P., Matadeen, R., Stoldt, M., Wohnert, J., Gorlach, M., van Hee, M., and Brimacombe, R. 2000. The $3 \mathrm{D}$ arrangement of the $23 \mathrm{~S}$ and $5 \mathrm{~S}$ rRNA in the Escherichia coli $50 \mathrm{~S}$ ribosomal subunit based on a cryo-electron microscopic reconstruction at 7.5 A resolution. J. Mol. Biol. 298: 35-59.

Napthine, S., Liphardt, J., Bloys, A., Routledge, S., and Brierley, I. 1999. The role of RNA pseudoknot stem 1 length in the promotion of efficient -1 ribosomal frameshifting. J. Mol. Biol. 288: 305320.

Nissen, P., Hansen, J., Ban, N., Moore, P.B., and Steitz, T.A. 2000. The structural basis of ribosome activity in peptide bond synthesis. Science 289: 920-930.

Nissen, P., Ippolito, J.A., Ban, N., Moore, P.B., and Steitz, T.A. 2001. RNA tertiary interactions in the large ribosomal subunit: The Aminor motif. Proc. Natl. Acad. Sci. 98: 4899-4903.

Nixon, P., Rangan, A., Kim, Y., Rich, A., Hoffman, D., Hennig, M., and Giedroc, D. 2002. Solution structure of a luteoviral p1-p2 frameshifting mRNA pseudoknot. J. Mol. Biol. 322: 621-633.

Noller, H.F., Yusupov, M.M., Yusupova, G.Z., Baucom, A., and Cate, J.H. 2002. Translocation of tRNA during protein synthesis. FEBS Lett. 514: 11-16.

Ogle, J.M., Brodersen, D.E., Clemons Jr., W.M., Tarry, M.J., Carter, A.P., and Ramakrishnan, V. 2001. Recognition of cognate transfer RNA by the 30S ribosomal subunit. Science 292: 897-902.

Pestova, T.V., Borukhov, S.I., and Hellen, C.U.T. 1998. Eukaryotic ribosomes require initiation factors 1 and $1 \mathrm{~A}$ to locate initiation codons. Nature 394: 854-859.

Pioletti, M., Schlunzen, F., Harms, J., Zarivach, R., Gluhmann, M., 
Avila, H., Bashan, A., Bartels, H., Auerbach, T., Jacobi, C., et al. 2001. Crystal structures of complexes of the small ribosomal subunit with tetracycline, edeine and IF3. EMBO J. 20: 18291839.

Polacek, N., Gaynor, M., Yassin, A., and Mankin, A.S. 2001. Ribosomal peptidyl transferase can withstand mutations at the putative catalytic nucleotide. Nature 411: 498-501.

Rice, N.R., Stephens, R.M., Burny, A., Gilden, R.V. 1985. The gag and pol genes of Bovine Leukemia Virus: Nucleotide sequence and analysis. Virology 142: 357-377.

Schluenzen, F., Tocilj, A., Zarivach, R., Harms, J., Gluehmann, M., Janell, D., Bashan, A., Bartels, H., Agmon, I., Franceschi, F., et al. 2000. Structure of functionally activated small ribosomal subunit at 3.3 angstroms resolution. Cell 102: 615-623.

Schmeing, T.M., Seila, A.C., Hansen, J.L., Freeborn, B., Soukup, J.K., Scaringe, S.A., Strobe, S.A., Moore, P.B., and Steitz, T.A 2002. A pre-translocational intermediate in protein synthesis observed in crystals of enzymatically active 50 S subunits. Nat. Struct. Biol. 9: $225-230$.

Simonson, A.B. and Lake, J.A. 2002. The transorientation hypothesis for codon recognition during protein synthesis. Nature 416: 281285.

Somogyi, P., Jenner, A.J., Brierley, I.A., and Inglis, S.C. 1993. Ribosomal pausing during translation of an RNA pseudoknot. Mol. Cell. Biol. 13: 6931-6940.

Spahn, C.M., Beckmann, R., Eswar, N., Penczek, P.A., Sali, A., Blobel, G., and Frank, J. 2001. Structure of the 80 S ribosome from Saccharomyces cerevisiae - tRNA-ribosome and subunit-subunit interactions. Cell 107: 373-386.

Stahl, G., McCarty, G.P., and Farabaugh, P.J. 2002. Ribosome structure: Revisiting the connection between translational accuracy and unconventional decoding. Trends Biochem. Sci. 27: 178-183.

Su, L., Chen, L., Egli, M., Berger, J.M., and Rich, A. 1999. Minor groove RNA triplex in the crystal structure of a ribosomal frameshifting viral pseudoknot. Nat. Struct. Biol. 6: 285-292.
Thompson, J., Kim, D.F., O'Connor, M., Lieberman, K.R., Bayfield, M.A., Gregory, S.T., Green, R., Noller, H.F., and Dahlberg, A.E. 2001. Analysis of mutations at residues A2451 and G2447 of $23 \mathrm{~S}$ rRNA in the peptidyltransferase active site of the 50S ribosomal subunit. Proc. Natl. Acad. Sci. 98: 9002-9007.

$\mathrm{Tu}, \mathrm{C}$., Tzeng, T-H., and Bruenn, J.A. 1992. Ribosomal movement impeded at a pseudoknot required for ribosomal frameshifting. Proc. Natl. Acad. Sci. 89: 8636-8640.

Tumer, N.E., Parikh, B., Li, P., and Dinman, J.D. 1998. Pokeweed antiviral protein specifically inhibits $\mathrm{Ty} 1$ directed +1 ribosomal frameshifting and Ty1 retrotransposition in Saccharomyces cerevisiae. J. Virol. 72: 1036-1042.

Wang, Y., Wills, N.M., Du, Z., Rangan, A., Atkins, J.F., Gesteland, R.F., and Hoffman, D.W. 2002. Comparative studies of frameshifting and nonframeshifting RNA pseudoknots: A mutational and NMR investigation of pseudoknots derived from the bacteriophage T2 gene 32 mRNA and the retroviral gag-pro frameshift site. RNA 8: 981-996

Wang, Z., Gaba, A., and Sachs, M.S. 1999. A highly conserved mechanism of regulated ribosome stalling mediated by fungal arginine attenuator peptides that appears independent of the charging status of arginyl-tRNAs. J. Biol. Chem. 274: 37565-37574.

Weiss, R.B., Dunn, D.M., Shuh, M., Atkins, J.F., and Gesteland, R.F. 1989. E. coli ribosomes re-phase on retroviral frameshift signals at rates ranging from $2 \%$ to $50 \%$. New Biol. 1: 159-169.

Wimberly, B.T., Brodersen, D.E., Clemons Jr., W.M., Morgan-Warren, R.J., Carter, A.P., Vonrhein, C., Hartsch, T., and Ramakrishnan, V. 2000. Structure of the $30 \mathrm{~S}$ ribosomal subunit. $\mathrm{Na}-$ ture 407: 327-339.

Yusupov, M.M., Yusupova, G.Z., Baucom, A., Lieberman, K., Earnest, T.N., Cate, J.H., and Noller, H.F. 2001. Crystal structure of the ribosome at 5.5 A resolution. Science 292: 883-896.

Yusupova, G.Z., Yusupov, M.M., Cate, J.H., and Noller, H.F. 2001. The path of messenger RNA through the ribosome. Cell 106: 233 241. 

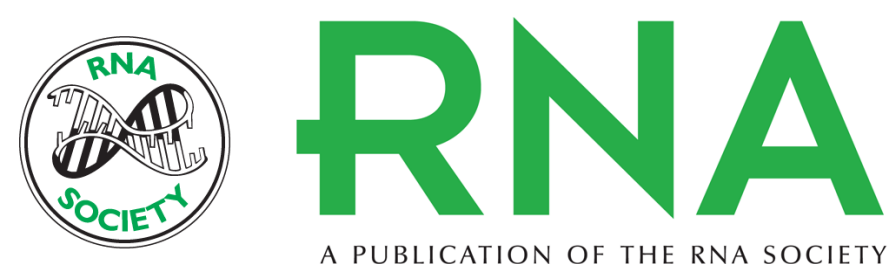

A PUBLICATION OF THE RNA SOCIETY

\section{The 9-Å solution: How mRNA pseudoknots promote efficient programmed -1 ribosomal frameshifting}

EWAN P. PLANT, KRISTI L. MULDOON JACOBS, JASON W. HARGER, et al.

RNA 2003 9: 168-174

References This article cites 59 articles, 24 of which can be accessed free at: http://rnajournal.cshlp.org/content/9/2/168.full.html\#ref-list-1

\section{License}

Email Alerting Receive free email alerts when new articles cite this article - sign up in the box at the Service top right corner of the article or click here. 